\title{
Referrals to a secondary care dental clinic for anxious adult patients: implications for treatment
}

\author{
P. McGoldrick, ' J. Levitt, ${ }^{2}$ A. de Jongh, ${ }^{3}$ A. Mason, ${ }^{4}$ and D. Evans, ${ }^{5}$
}

\begin{abstract}
Objectives This study aimed to determine the methods suggested by general dental practitioners for management of patients with dental anxiety whom they refer to a dental hospital setting, the treatment modalities eventually used with such patients and the relationship between patients previous sedation experience and the current referral.

Methods Consecutive referral letters $(n=125)$ for management of patients with dental anxiety over a 16 month period were analysed for content, including reason for referral and suggested treatment modalities. Patient records were also examined for previous sedation experience.

Results From 115 referrals eligible for analysis, the dentists requested management of anxiety using pharmacological methods in 113 referrals with only two referrals mentioning psychologically-based treatments. In secondary care, $29 \%$ of the adult referrals opted for dental treatment using psychological techniques alone.

Conclusions In spite of the efficacy of psychological treatments for dental anxiety, primary and secondary care dentists appear not to be suggesting or promoting their use for patients with dental anxiety. Further research into the availablility of, and barriers to accessing the full range of services for those with dental anxiety, including patient perspectives, needs to be undertaken.
\end{abstract}

\begin{abstract}
$\mathrm{D}$ ental fear can be a major barrier to completion of dental treatment in primary care. Patients fearful of impending invasive procedures often present only when in pain. Then effective pain control and satisfactory treatment completion can be difficult for the dental practitioner and patient. ${ }^{1}$ For the already anxious patient, this can result in further avoidance leading to poorer oral health. Anxiety can arise from two main sources: a previous direct negative experience or indirectly, through vicarious information. Irrespective of the cause, a high level of dental anxiety can prevent dental teatment from being completed by the dental practitioner. The true extent of the problem is unknown as many of those with difficulties either avoid dental treatment or attend only for sympto-
\end{abstract}

\footnotetext{
$1^{*}$ Lecturer in Health Psychology/Clinical Psychologist, University of Dundee Dental School; ${ }^{2}$ Staff Grade Dentist / Honorary Clinical Lecturer, University of Dundee Dental School; ${ }^{3}$ Associate Professor, ACTA, University of Amsterdam, Louwesweg 1, 1066 EA Amsterdam, The Netherlands; ${ }^{4}$ Lecturer, University of Dundee Dental School; ${ }^{5}$ Senior Lecturer/Honorary Consultant, University of Dundee Dental School

${ }^{*}$ Correspondence to: Pauline McGoldrick, Dental Public Health and Health Psychology, University of Dundee Dental School, Park Place, Dundee DD1 4HR Email:p.m.mcgoldrick@dundee.ac.uk

REFEREED PAPER

Received 07.12.00; Accepted 16.07.01

(C) British Dental Journal 2001; 191: 686-688
}

matic care, but figures are available from some countries. In the 1998 United Kingdom Adult Dental Health Survey, 31\% of dentate adults (23\% men and $40 \%$ women) reported being definitely nervous of some types of dental treatment. ${ }^{1}$ Many children are also fearful of dental treatment with figures ranging from $6.7 \%$ in Swe$\mathrm{den}^{2}$ to $19.5 \%$ in America. ${ }^{3,4}$ Treatment services for people with dental anxiety are disjointed in many countries with formal registration unnecessary. A recent review of services in Scotland identified lack of data about dentally anxious patients, lack of understanding of the psychology of anxiety and lack of information regarding services available to the anxious patient.$^{5}$

When dental treatment difficulties arise, dental practitioners often refer patients to secondary care facilitites such as a dental hospital or a specialist clinic. In such places, a wide range of treatment options may be available for the anxious patient including medication and psychological therapies. Pharmacological or sedation techniques may include oral medication, inhalation sedation with nitrous oxide, intravenous sedation with one or more sedative drugs or general anaesthesia. For psychological intervention methods, behavioural therapy in the form of in vivo exposure (that is graded and prolonged exposure to the phobic stimulus) is the treatment of choice for specific, uncomplicated phobias. ${ }^{6}$ Other approaches to treat dental anxiety which show good long-term effectiveness include systematic desensitization, ${ }^{7}$ relaxation and distraction, ${ }^{8}$ hypnosis, ${ }^{9}$ imaginal exposure,${ }^{10}$ cognitive restructuring, ${ }^{11}$ and Eye Movement Desensitization and Reprocessing (EMDR). ${ }^{12}$

The General Dental Council has recommended in its most recent publication on the dental undergraduate curriculum The First Five Years that dental students be taught the value of behavioural methods of anxiety management. ${ }^{13}$ To develop teaching in this area a dental anxiety clinic for clinical teaching of anxiety management techniques to dental undergraduates was initiated at Dundee Dental Hospital and School (DDH) in October 1998. The clinic, staffed by a clinical psychologist, two dentists and a dental assistant offers a minimum of five clinical sessions to each final year dental undergraduate student after completion of a structured training programme. One difficulty which arose was that adult patients who were referred to DDH with a history of problems accepting regular dental care due to anxiety appeared to be referred for intravenous sedation primarily. This was confirmed to be a long standing pattern of referring by the dentist previously responsible for treating these patients and was clearly a barrier to offering suitable patients an alternative treatment option which did not involve sedation. It was thus considered important to investigate this observed phenomenon empirically.

Hence, we set out to investigate referrals of patients with dental anxiety who had been referred to the DDH during the study period. The study had three aims: 1) to determine the methods suggested by general dental practitioners for management of patients with dental anxiety whom they refer to a dental hospital setting, 2) to determine which treatment modalities are eventually used with such patients 
within secondary care and 3) to determine if there is a relationship between patients' previous sedation experience and the current referral.

\section{Methods}

\section{Sample}

Referral letters and hospital dental records of 125 consecutive patients referred during November 1996 and March 1998 were surveyed. Ten were excluded due to failure to attend the clinic assessment appointment. None of the 10 referrals excluded mentioned a non-pharmacological approach to management of the patient's anxiety. Therefore, 21 men and 94 women with a mean age of 34 years $(\mathrm{SD}=10.4$, range $=18-62)$ were included in the study.

Design and procedure

A retrospective study design was used. Letters referring patients specifically for management of dental anxiety were scrutinised for treatment modality requested by the referring dentist. Previous experience of intravenous sedation while undergoing dental procdures and eventual treatment modality used in the dental hospital setting were also recorded from the patient's dental records.

Data analysis

Data were analysed using chi-squared statistics. Of the 125 patient referrals scrutinised, $8 \%(n=10)$ failed to attend for the initial appointment and were thus excluded from the analyses.

\section{Results}

All referral letters specified a treatment modality for the management of the patient's anxiety. The majority of the referrers were 77 general dental practitioners (67\%), while interdepartmental referrals accounted for $29 \%(\mathrm{n}=33)$ and the remaining $4 \%$ were self referrers $(n=5)$. Of the 115 patients who attended for initial examination, only two of the patient referral letters suggested the use of non-pharmacological management of the patient's anxiety; both came from general dental practitioners. The remaining 113 referral letters requested pharmacological management. Of those dentists requesting pharmacological approaches, 109 requested intravenous sedation, 3 requested general anaesthesia and 1 requested inhalation sedation. The anxiety treatment modalities eventually used with the patients while undergoing dental procedures were mainly intravenous sedation $(n=71)$ followed by behavioural techniques $(n=34)$, general anaesthesia $(n=7)$ and inhalation sedation $(n=3)$. The frequency of patients that actually received behavioural treatment was (x32) higher than the frequency of patients that were referred for this mode of treatment (Table 1).

Table I. Treatments requested by referrer compared with treatments used in secondary care clinic.

\begin{tabular}{|c|c|c|c|c|c|}
\hline \multirow{3}{*}{$\begin{array}{l}\text { Treatments } \\
\text { requested (n) } \\
\text { IV sedation }\end{array}$} & \multicolumn{5}{|c|}{ Treatments used (n) } \\
\hline & IV sed & GA & IHS & Behavioural & Total \\
\hline & 69 & 7 & 2 & 31 & 109 \\
\hline GA & I & 0 & 0 & 2 & 3 \\
\hline IHS & 0 & 0 & 1 & 0 & 1 \\
\hline Behavioural & I & 0 & 0 & I & 2 \\
\hline Total & 71 & 7 & 3 & 34 & 115 \\
\hline
\end{tabular}

Sixty-five percent $(n=75)$ of this patient group had at least one previous experience of dental treatment using intravenous sedation in the hospital setting. There were 14 patients who had more than 6 previous referrals for dental treatment with intravenous sedation (with a maximum of 21). The patients who had no previous experience of intravenous sedation were more likely than those who had at least one previous experience of sedation, to accept dental treatment on this occasion using behavioural management techniques (Table 2). Using chi-squared with Yates Correction for Continuity, there was no significant association $\left(\chi^{2}=1.28, \mathrm{df}(1), \mathrm{p}>0.05\right)$ between patients' previous sedation experience and use of behavioural techniques for current treatment.

Table 2. Previous sedation treatments by treatment used in secondary care clinic.

\begin{tabular}{|c|c|c|c|c|c|}
\hline \multirow{3}{*}{$\begin{array}{l}\text { Previous } \\
\text { IV Sedation (n) } \\
0 \\
\text { I or greater }\end{array}$} & \multicolumn{5}{|c|}{ Treatment Used (n) } \\
\hline & IV sedation & GA & $\mathrm{IHS}$ & Behav & Total \\
\hline & $\begin{array}{l}36 \\
35\end{array}$ & $\begin{array}{l}2 \\
5\end{array}$ & $\begin{array}{l}1 \\
2\end{array}$ & $\begin{array}{l}21 \\
13\end{array}$ & $\begin{array}{l}60 \\
55\end{array}$ \\
\hline Total & 71 & 7 & 3 & 34 & 115 \\
\hline
\end{tabular}

\section{Discussion and conclusions}

In spite of the efficacy of psychological treatments for dental anxiety, dentists in this sample rarely suggested the use of such treatment methods in their referral letters to a secondary care clinic. One possible explanation is that dental practitioners may not be aware that such treatment methods are available. This is in contrast to countries like the Netherlands, where special dental fear clinics are commonly known to both the general public and to general dental practitioners. ${ }^{14}$

One option that can be offered to patients is behaviour therapy (that is exposure to the feared stimulus) which has been found to be the most effective treatment for specific phobias, including dental phobia. A recent study by Aartman and colleagues found that behavioural therapy was effective for $46.5 \%$ of patients who attended a dental fear clinic for dental treatment. ${ }^{15}$ Compared with 'easier' alternatives such as intravenous sedation or general anaesthesia, a behavioural approach requires commitment, engagement and courage from patients, which many may find difficult to consider. However, it is important to note that in many situations the application of these alternatives is only a short-term solution. In the long run patients remain anxious, may continue to avoid necessary dental care and have to be treated again with help of pharmacological methods. This notion is supported by the finding of this study that for the majority of the patients in this study it was not the first time that they were referred for pharmacological treatment. Obviously with new guidance on the use of general anaesthesia, particularly in children, there has been a paradigm shift to less risky methods. However, there may be a danger that the use of intravenous sedation will remain the treatment of choice for undertaking dental procedures in those who are anxious, in spite of the absence of good clinical evidence for its efficacy in terms of anxiety reduction.

Almost half of the sample had previously undergone dental treatment using intravenous sedation on one or more occasions. This lends some support to the cyclical nature of sedation techniques. Given that dental practitioners' requests often have the greatest influence on decision-making in the dental surgery and will likely have discussed the referral with the patient and gained consent, patients referred for treatment will often attend expecting and prepared for only that method. This can make it difficult for those in specialist secondary care to offer different treatment options if deemed in the best interests of the patient.

Of course, it can be argued that many of these adult patients display long term neglect in their dentition, which often requires multiple extractions and extensive restorative work justifying intravenous sedation. Therefore, in many cases providing intial dental treatment using pharmacological techniques may be the best option. However, once the dentition is stabilised, residual and future dental treatment should be targeted using psychological treatments which can increase the patient's own sense of self-effi- 
cacy and build confidence to cope with future dental procedures. One third of the patients in this study opted for behavioural techniques when offered the option by one hospital dentist, suggesting that this treatment approach may be an acceptable and suitable option for many patients if given the choice. If patients are not given such options in primary care, it is likely that the cyclical burden of dependence on methods such as intravenous sedation will continue to grow. Patients will not have the opportunity to expose themselves in vivo to procedures due to amnesic effects of the drugs given during intravenous sedation which may prevent habituation (anxiety reduction) from taking place..$^{15}$

The conclusions that can be drawn from this study are limited by the retrospective design, the size of the sample and the specialised setting chosen. It is not known if similar referral patterns occur in other parts of the UK, either in dental schools or other specialist centres for treating dental anxiety. Practising dentists will recognise that they have a duty to offer patients all treatment options available (including behavioural techniques for which there is proven effectiveness) and to assign their patients to the most appropriate treatment modality for them to meet their individual needs. ${ }^{15}$ Clinical psychologists accept referrals from a wide range of agencies and health professionals. Referring patients with dental anxiety problems is an example of an appropriate referral. However, the demand for generic clinical psychology services may mean that accessing treatment involves a lengthy waiting time unless dedicated funding is available. Guidance for the dental professional interested in purchasing clinical psychology services has been produced. ${ }^{16}$ Further research is required about the other barriers that prevent some treatments from being considered, the psychology of dental anxiety including categorisation of patients using standard measures and preferred treatment options of patients in the UK, as these appear to be lacking ${ }^{5}$
1. Walker A, Cooper I. Adult Dental Health Survey: Oral Health in the UK 1998. London: Stationery Office, 2000.

2. Klingberg G, Berggren U, Noren J G. Dental fear in an urban Swedish child population: prevalance and concomitant factors. Community Dent Health 1994; 11: 208-214.

3. Milgrom P, Fislet L, Melrick S, Weinstein P. The prevalance and practice management consequences of dental fear in a major US city. J Am Dent Assoc 1988; 116: 641-647.

4. Raadal M, Milgrom P, Weinstein P, Mancl L, Cauce A M. The Prevalence of Dental Anxiety in Children from Low-Income Families and its Relationship to Personality Traits. J Dent Res 1995; 74: 1439-1443.

5. National Dental Advisory Committee. Services for the dentally anxious. Scotland: National Dental Advisory Committee, 1998.

6. Emmelkamp P, Bouman T, Scholing A. Anxiety Disorders: A Practitioner's Guide. Chichester: Wiley \& Sons, 1992.

7. Getka E J, Glass C R. Behavioural and Cognitive-Behavioural Approaches to the Reduction of Dental Anxiety. Behav Ther 1992; 23: 433-448.

8. Corah N L, Gale E N, IIling S J. The use of relaxation and distraction to reduce psychological stress during dental procedures. J Am Dent Assoc 1979; 98: 390-394.

9. Peretz B. Confusion as a technique to induce hypnosis in a severely anxious pediatric patient. J Clin Pediatr Dent 1999b; 21: 27-30.

10. Horowitz L G. Audiotaped relaxation, implosion, and rehearsal for the treatment of patients with dental phobia. Gen Dent 1992; 40: 242-247.

11. Levitt J, McGoldrick P, Evans D. The management of severe dental phobia in an adolescent boy: A case report. Int J Pediatr Dent 2000; 11: 208-214.

12. DeJongh A, van den Oord H J M, ten Broeke, E. Efficacy of Eye Movement Desensitization and Reprocessing (EMDR) in the treatment of specific phobias: Four single-case studies on dental phobia. J Clin Psychol. In Press.

13. General Dental Council. The First Five Years: The dental undergraduate curriculum. London: General Dental Council, 1997.

14. Aartman I H, de Jongh A, Makkes P C, Hoogstraten J. Treatment modalities in a dental fear clinic and the relation with general psychopathology and oral health variables. Br Dent J 1999; 186: 467-471.

15. Thompson J M, Neave N, Moss M C, Scholey A B, Wesnes K, Girdler N. Cognitive properties of sedation agents: comparison of the effects of nirtous oxide and midazolam on memory and mood. Br Dent J2000; 187: 557-562.

16. Lindsay S. Clinical Psychology in Dentistry: A Guide to Purchasers of Clinical Psychology Services. London: British Psychological Society, 1996. Report No.: Briefing Paper No. 11. 